\title{
Le télescope Canada-France-Hawaï sur la piste de l'énergie noire
}

Vanina Ruhlmann-Kleider (vanina.ruhlmann-kleider@cea.fr)

Service de Physique des Particules de I'Irfu au CEA/Saclay, 91191 Gif-sur-Yvette Cedex

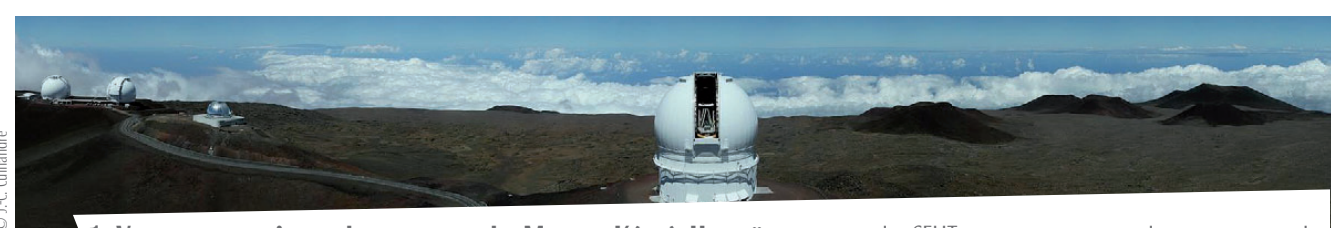

1. Vue panoramique du sommet du Mauna Kéa à Hawaï montrant le (FHT au centre, coupole ouverte, et les télescopes IRTF (Infrared Telescope Facility), Keck I et II à gauche.

Les supernovæ, explosions

d'étoiles en fin de vie, sont

parmi les phénomènes les

plus lumineux de l'Univers.

Certaines de ces explosions,

dites de « type Ia », ont

même une luminosité

quasi reproductible.

Mesurer leur flux revient

donc à mesurer leur distance.

Or, les mesures faites sur

les supernovæ Ia lointaines

s'accordent toutes :

elles sont moins lumineuses,

et donc plus lointaines,

qu'attendu.

Ainsi, l'expansion de l'Univers,

au lieu de ralentir, s'accélère.

Retour sur l'une des plus

grandes surprises de la

cosmologie moderne,

confirmée sans ambiguïté

par le récent relevé de

supernovæ lointaines

du télescope Canada-France-

Hawaï, le « Supernova

Legacy Survey ».

\section{Du Big Bang à l'expansion accélérée de l'Univers}

Comme pour le reste de la physique, le $\mathrm{XX}^{\mathrm{e}}$ siècle a fait faire un pas de géant à la cosmologie. Notre vision des lois qui gouvernent l'Univers s'en est trouvée considérablement modifiée. Le début du siècle a vu les mesures de distance s'affiner et les astronomes réaliser que certains objets lumineux sont situés en dehors de notre propre galaxie. En 1929, Edwin Hubble étudie les spectres des galaxies proches de la nôtre et remarque qu'ils sont décalés vers les plus grandes longueurs d'onde (on parle de « décalage spectral vers le rouge "), preuve que les galaxies s'éloignent les unes des autres à une vitesse proportionnelle à la distance qui les sépare. L'idée d'un Univers en expansion émerge et trouve naturellement sa place dans le modèle dit du Big Bang, qui fait naître l'Univers d'une singularité initiale, que l'on peut voir comme l'explosion d'un grain de matière dense et chaud. Associé à la relativité générale d'Einstein et à la physique nucléaire, le modèle permet de décrire les grandes étapes de l'évolution ultérieure de l'Univers.

Après l'explosion initiale, celui-ci se dilate régulièrement et se refroidit, rendant possible la synthèse des premiers noyaux atomiques, hydrogène et hélium, qui se fait essentiellement dans les trois premières minutes après le Big Bang. S'ensuit une période d'équilibre entre matière et rayonnement, durant laquelle le rayonnement détruit les édifices atomiques plus complexes aussitôt qu'ils sont créés par la matière. Après 380000 ans environ, l'Univers s'est suffisamment refroidi et la formation des atomes n'est plus contrariée par le rayonnement. Dès lors, matière et rayonnement suivent des évolutions différentes. Ainsi, le rayonnement, libéré, peut traverser l'Univers. L'existence d'un tel rayonnement, dit «fossile» est l'une des prédictions fortes du modèle du Big Bang. Elle recevra une confirmation éclatante lors de la découverte fortuite $\mathrm{du}$ fond diffus cosmologique en 1964 par A. Penzias et R. Wilson, lors de la mise au point d'antennes de radioastronomie de haute sensibilité. Quelques années plus tard, une autre prédiction du modèle sera également couronnée de succès : les abondances prédites pour les éléments légers produits lors de la nucléosynthèse primordiale évoquée plus haut sont en accord avec les observations. À la fin des années 70, le modèle du Big Bang s'impose donc comme le cadre théorique le plus crédible pour décrire l'évolution de l'Univers.

Loin s'en faut, pourtant, que tous les détails soient connus. Ainsi, dans les années 30, les premières mesures de masse des amas de galaxies montrent un désaccord selon que la masse de l'amas est estimée d'après son influence gravitationnelle ou d'après sa luminosité. Depuis les années 70, les observations se diversifient et se multiplient, mais toutes vont dans le même sens. Il est nécessaire de supposer l'existence dans l'Univers d'une matière dite noire, différente de la matière ordinaire et qui ne donne aucun signal électromagnétique. Depuis le début des années 90, les expériences de cosmologie observationnelle, par exemple celles qui étudient le rayonnement fossile, sont capables de mesurer précisément les densités correspondant aux deux types de matière. Leurs réponses sont concordantes : la matière ordinaire ne représente que $16 \%$ environ de la densité totale de matière dans l'Univers, la matière noire est cinq fois plus importante.

Le $\mathrm{XX}^{\mathrm{e}}$ siècle s'est achevé sur une autre surprise de taille pour les cosmologistes. À cause de l'attraction universelle, la dilatation de l'Univers devrait se ralentir. Or, à la fin des années 90, les premières observations de supernovæ de type Ia 
Image de supernova prise au télescope Canada-France-Hawaï par la caméra Megacam, dans une région du ciel différente de celles observées par SNLS. II s’agit de IC443, résidu de supernova de type gravitationnel, situé dans notre galaxie dans la constellation des Gémeaux.

lointaines - situées à plusieurs milliards d'années-lumière - révèlent que le flux apparent de ces objets est plus faible que ce que l'on attendrait pour un univers composé exclusivement de matière (ordinaire ou noire). Il semble que la distance parcourue par les photons émis par les supernovæ soit plus grande que prévu. Il faut alors admettre l'existence dans l'Univers d'une composante énergétique qui ne soit ni matière ni rayonnement, mais qui, à l'inverse de l'attraction universelle, accélère l'expansion. D'un point de vue théorique, une telle composante peut se déduire des équations d'Einstein de la Relativité Générale à condition d'y rajouter une simple constante, appelée constante cosmologique. Mais d'autres descriptions théoriques lui donnent un contenu plus fondamental. Dans l'état actuel des observations, il n'est pas possible de trancher et cette composante, inscrite depuis au bilan énergétique de l'Univers, a reçu le nom d' "énergie noire ». Sa nature demeure donc inconnue. Par contre, sa densité, déduite des mesures cosmologiques, domine l'Univers actuel, puisqu'elle représente environ trois quarts du contenu énergétique de l'Univers, contre un quart pour la matière. Dans ces conditions, l'expansion de l'Univers au lieu de ralentir, s'accélère.

\section{Le relevé de supernovæ du CFHT}

Ce résultat était si inattendu que de nouveaux projets d'étude de supernovæ lointaines ont été lancés. Le Supernova Legacy Survey (SNLS) appartient à cette seconde génération d'expériences. Il en est également le fleuron. SNLS utilise le télescope Canada-France-Hawai de 3,6 m de diamètre situé sur le sommet du Mauna Kéa à Hawaï (fig. 1). Programmé pour cinq ans, de 2003 à 2008, le relevé affichait l'ambition de détecter et mesurer précisément plusieurs centaines de supernovæ de type Ia (SN Ia), à comparer à une cinquantaine d'objets étudiés par les expériences antérieures.
Les SN Ia (voir encadré) sont des phénomènes rares, typiquement une explosion par siècle pour une galaxie comme la nôtre. L'efficacité de détection est donc le premier facteur important pour un relevé de supernovæ. Pour ce faire, SNLS bénéficie de la caméra à grand champ Megacam, caméra CCD de 340 millions de pixels. Instrument unique au monde, son champ de 1 degré carré (l'équivalent de quatre fois la pleine lune) permet d'observer en un seul pointé de grandes portions du ciel, susceptibles de contenir simultanément jusqu'à une dizaine de supernovæ. Des poses longues permettent de détecter des SN Ia suffisamment lointaines pour tester des distances de plusieurs milliards d'années-lumière. Afin d'assurer la permanence des détections tout au long de l'année, les observations sont faites sur quatre champs de 1 degré carré chacun, chaque champ étant observable depuis Hawai sur une durée de 7 mois environ. SNLS suit donc deux champs simultanément à toute époque de l'année. Les supernovæ y sont détectées en recherchant une augmentation de flux par rapport à celui des galaxies qui les hébergent, comme sur l'exemple de la figure 2 .

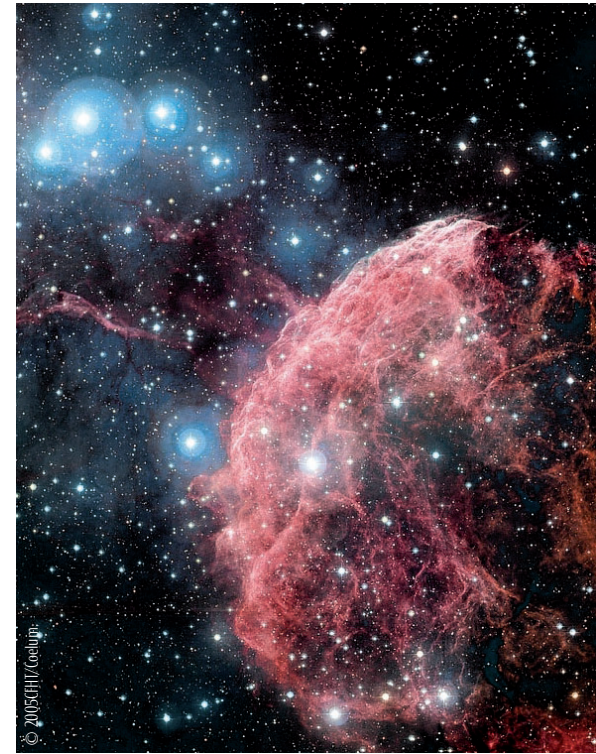

Les SN Ia sont des phénomènes très lumineux mais transitoires. L'émission de lumière qui les accompagne présente un profil temporel typique, appelé courbe de lumière, croissant sur une vingtaine de jours après l'explosion, pour décroître et finir par s'évanouir au bout d'une cinquantaine de jours (fig. 3). Il est important de mesurer précisément la courbe de lumière de tous les candidats supernovæ détectés. Pour ce faire, SNLS bénéficie d'une stratégie de suivi optimale. Rester en permanence sur les mêmes champs tant que ceux-ci sont visibles permet de suivre dans le temps tous les candidats détectés. Les mesures de flux sont réalisées régulièrement tous les trois à quatre jours, en dehors des périodes de pleine lune. Quatre bandes de fréquence sont utilisées, de l'optique à l'infrarouge proche pour couvrir toute la gamme de longueurs d'onde du spectre des supernovæ (fig. 3). Les courbes de lumière obtenues sont très bien échantillonnées en temps, ce qui constitue une amélioration notable par rapport aux programmes antérieurs, qui

\section{Les supernovæ de type Ia : des chandelles standard}

Les supernovæ de type la (SN la) observées jusqu'à ce jour ont des propriétés spectrales et lumineuses très homogènes. Le mécanisme imaginé pour rendre compte de ce fait est le suivant. Les SN la seraient dues à l'explosion thermonucléaire d'une naine blanche, étoile en fin de vie, accrétant la matière d'une étoile compagne géante. La naine blanche gagne en masse jusqu'à approcher la limite dite de Chandrasekhar. La température interne de l'étoile s'est alors suffisamment élevée pour que se déclenche une combustion nucléaire explosive : les éléments du cœur de l'étoile, essentiellement du carbone et de l'oxygène, brûlent en donnant du $56 \mathrm{Ni}$, et cette combustion dégage une énergie telle que l'étoile finit par exploser. C'est la désintégration ultérieure du ${ }^{56} \mathrm{Ni}$ en ${ }^{56} \mathrm{Co}$ puis en ${ }^{56} \mathrm{Fe}$ qui définit la luminosité de la supernova et rend I'objet aussi brillant que quelques milliards de soleils, l'équivalent d'une petite galaxie. Comme la masse de l'étoile, et donc la quantité de nickel produite, est pratiquement toujours la même lors de l'explosion, les SN la présentent des luminosités similaires.

De ce fait, on peut les utiliser comme des « chandelles standard » pour mesurer des distances, car leur flux apparent (i.e. mesuré) ne dépend que de la distance parcourue par les photons entre l'instant de l'explosion et celui de l'observation. 


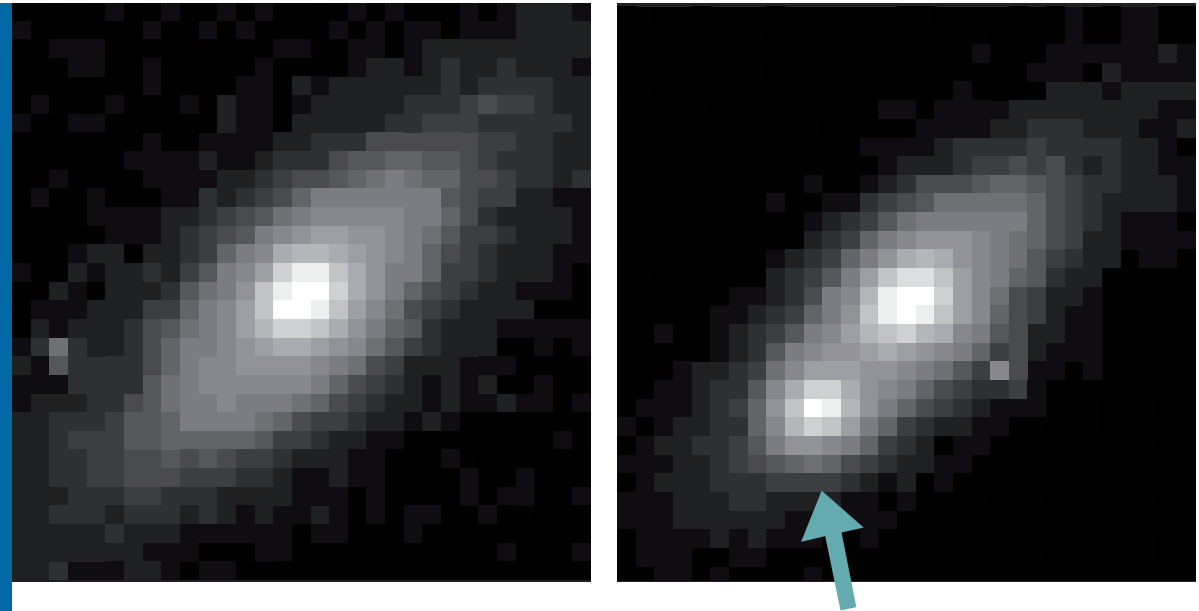

2. À gauche : image (grossie) d'une galaxie vue par SNLS avant explosion de la supernova 04D1dc; à droite : image de cette même galaxie après explosion. l'accroissement de flux apporté par la supernova (25\% par rapport au flux galactique sous-jacent) est clairement visible au bord de la galaxie (indiqué par une flèche en bas à gauche sur le cliché de droite).

\section{Références}

1 - http://en.wikipedia.org/wiki/Redshift \#Expansion_of_space

2 • P. Astier et al., AEA 447 (2006) 31.

3 • A. Conley et al., AJ 132 (2006) 1707.

4 - M. Sullivan et al., ApJ 648 (2006) 868.

5 • D. Howell et al., ApJ 667 (2007) 37.

6 • T.J. Bronder et al., AEA 477 (2008) 717.

7 • R.S. Ellis et al., ApJ 674 (2008) 51.

8 • Z. Ivezic et al., astro-ph/0805.2366.

9 • «l'expansion de l'Univers, pourquoi elle accélère », Ciel et Espace 444 (mai 2007) 26 .

« Énergie sombre, le fantôme qui dilate l'Univers », Ciel et Espace $\mathbf{4 5 4}$ (mars 2008) 38.

« Énergie noire, l'étrange moteur de I'Univers ", La Recherche $\mathbf{4 2 2}$ (juillet 2008) 31.
$>>>$

suivaient les supernovæ à quelques semaines d'intervalle et dans deux bandes de fréquence seulement.

Par ailleurs, dès sa détection par SNLS, toute supernova potentielle proche de son maximum de lumière est observée par des spectrographes sur des télescopes de 8 à $10 \mathrm{~m}$ de diamètre afin de déterminer précisément son décalage spectral et son type, thermonucléaire ou gravitationnel. Le temps d'observation alloué à SNLS permet de réaliser ces mesures sur une large fraction (environ 60\%) des supernovæ détectées. La plupart des spectres sont mesurés par le Very Large Telescope (VLT) de l'observatoire européen austral au Paranal (Chili). Les autres mesures sont effectuées sur les télescopes Keck à Hawaï et sur les deux télescopes Gémini, l'un à Hawai et l'autre au Chili. L'examen des spectres, outre la mesure du décalage spectral, permet, par la présence ou l'absence de certaines raies de référence, de déterminer le type des supernovæ. Celles-ci peuvent être dues à une explosion thermonucléaire dans le cas du type Ia ou à un effondrement gravitationnel pour les supernovæ de type Ib, Ic ou II. Les supernovæ gravitationnelles ne sont pas exploitables pour la cosmologie, du fait de leur grande variabilité en luminosité d'un objet à l'autre. Elles constituent un bruit de fond pour la recherche de SN Ia, dont on s'affranchit grâce aux mesures spectrales. À l'issue du relevé en juin 2008, le nombre de supernovæ de type Ia détectées et suivies par SNLS, et confirmées par la spectroscopie s'élève à environ 500 , soit 10 fois plus que le lot accumulé par les expériences antérieures. L'objectif du projet est donc rempli.

\section{Cosmologie et supernovæ de type Ia}

Au-delà de la confirmation du type de supernova, les mesures spectrales permettent également de déterminer l'un des ingrédients clés pour remonter à la cosmologie, à savoir le décalage spectral $^{1}$ de chaque supernova, noté $z$. En effet, l'expansion de l'Univers affecte les mesures de distance ; une même distance - par exemple celle entre deux galaxies - mesurée à des instants différents donne des valeurs différentes, croissantes au cours du temps. Il en est de même pour la longueur d'onde d'un photon entre le moment de l'émission et celui de son observation : les raies spectrales d'un objet céleste en expansion sont décalées vers les plus grandes longueurs d'onde par rapport aux raies du spectre émis. On peut montrer [1] que ce décalage spectral, d'origine cosmique, est une mesure directe du facteur d'expansion de la taille de l'Univers entre l'instant de l'émission et celui de l'observation, soit $(1+z)$. Ainsi, SNLS est sensible à des SN Ia de décalage spectral compris entre 0,2 et 1,2. Ces supernovæ ont donc explosé lorsque l'Univers avait une taille réduite de 20 à $50 \%$ par rapport à sa taille actuelle, c'est-à-dire lorsque l'Univers était plus jeune de 2 à 8 milliards d'années environ. Les supernovæ détectées par SNLS viennent donc du passé lointain de l'Univers et leur lumière a franchi des distances considérables - plusieurs milliards d'annéeslumière - avant de nous parvenir.
1 - Le décalage spectral z est défini par : $z=\left(\lambda_{\text {obs }}-\lambda_{\text {émission }}\right) / \lambda_{\text {émission. }}$ Pour un phénomène observé à un décalage spectral $z$, on a la relation $: 1+\mathrm{z}=\mathrm{a}\left(\mathrm{t}_{\mathrm{obs}}\right) / \mathrm{a}\left(\mathrm{t}_{\text {émission }}\right)$ où $\mathrm{a}(\mathrm{t})$ représente le facteur d'échelle de l'Univers à l'instant $t$, qui permet de retrouver la distance d'un objet, $\mathrm{R}(\mathrm{t})$, connaissant ses coordonnées comobiles $\chi$ : $\mathrm{R}(\mathrm{t})=\mathrm{a}(\mathrm{t}) \chi$. Dans un univers en expansion, $\mathrm{a}(\mathrm{t})$ croit avec le temps.

2 - Il est d'usage en astrophysique d'exprimer les flux lumineux sur une échelle logarithmique inversée, dite échelle des magnitudes. Plus un objet est lumineux, plus faible sera sa magnitude.

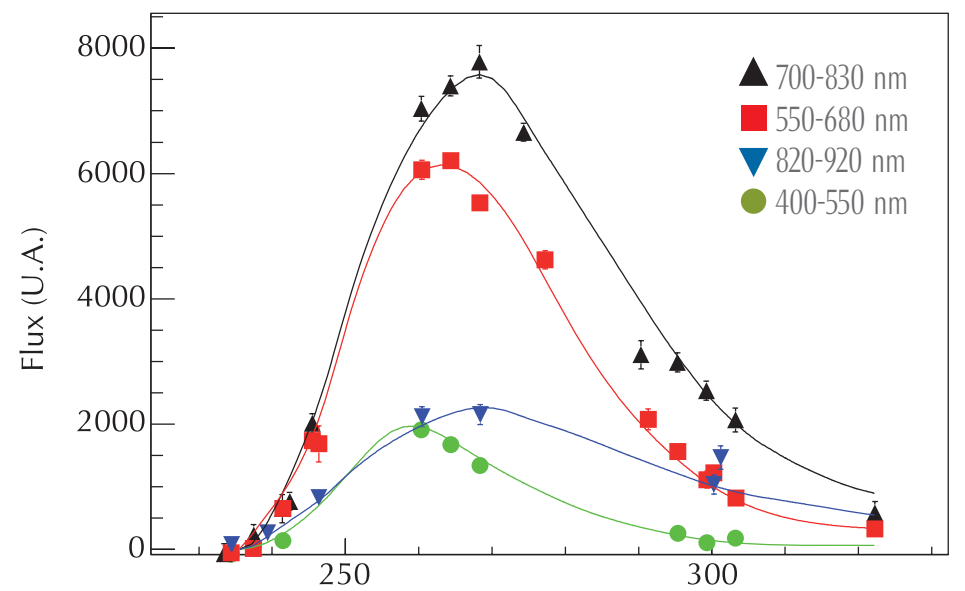

Nombre de jours depuis le 01/01/2003
3. Courbe de lumière de la supernova 03D4dh mesurée par SNLS. Les mesures sont effectuées dans quatre bandes passantes distinctes. La fréquence des observations permet d'avoir suffisamment de points de mesure pour remonter non seulement à la luminosité au pic, mais aussi à la forme globale de la courbe (temps de montée et de descente). 
4. Magnitude 2 apparente des SN Ia (déduite du flux lumineux au pic et corrigée des effets de dispersion intrinsèque) en fonction de leur décalage spectral. Les mesures de première année du SNLS (points), associées à des mesures sur des supernovæ proches (cercles), sont comparées aux prédictions pour un univers composé exclusivement de matière (tirets) et pour un univers en expansion accélérée, composé de $74 \%$ d'énergie noire et de $26 \%$ de matière (trait plein).

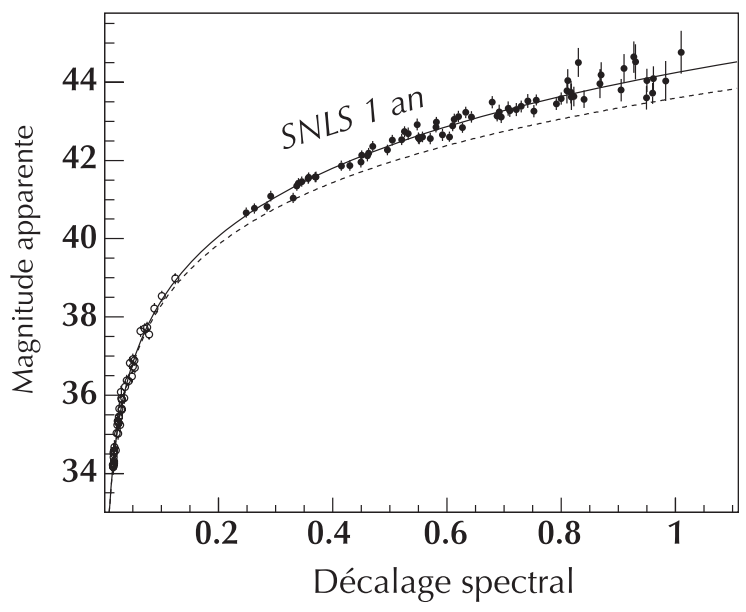

Or, sur de telles distances, le parcours des photons dépend significativement de l'évolution que l'Univers a suivie dans le passé. Ainsi, à décalage spectral donné, si l'on considère deux scénarios d'évolution conduisant à l'Univers actuel, l'un correspondant à un univers en expansion constante, l'autre à un univers en expansion accélérée, il faut un temps plus long si l'expansion est accélérée pour réaliser un même facteur d'expansion, i.e. un même décalage spectral. Or, un temps de parcours plus long implique une distance de parcours plus grande pour les photons, et donc un flux détecté plus petit. En effet, du fait de l'homogénéité des luminosités intrinsèques des SN Ia, le flux détecté mesure directement la distance parcourue par les photons depuis l'explosion. Plus la distance parcourue est grande, plus le flux détecté est faible. Mesurer le flux des SN Ia sur une gamme étendue de décalages spectraux doit donc permettre de remonter à l'évolution passée de l'Univers, elle-même déterminée par son contenu en matière et en énergie.

Cette analyse a été entreprise par SNLS à l'issue de la première année d'observations, sur la base de 70 SN Ia confirmées par la spectroscopie, soit autant que le lot accumulé jusqu'alors sur une dizaine d'années par les autres expériences. Les mesures, représentées sur la figure 4, montrent que, à décalage spectral donné, le flux reçu des SN Ia lointaines est plus faible que ce que l'on attendrait pour un univers dominé par la matière [2]. Les mesures sont, en revanche, en accord avec ce que l'on attend pour un univers en expansion accélérée, dominé par l'énergie noire. Le résultat de la première génération d'expériences de supernovæ lointaines est donc confirmé sans ambiguité. Mais les mesures réalisées par SNLS permettent d'aller plus loin et de préciser le contenu énergétique actuel de l'Univers. La matière n'en constitue que $26 \%$ (à une incertitude relative près de $16 \%)$, les $74 \%$ restants étant attribués à l'énergie noire. Les données de SNLS, couplées à celles d'autres observations cosmologiques, permettent aussi, et pour la première fois, de tester l'évolution temporelle de la densité d'énergie noire. Celle-ci se révèle constante dans le temps, à 10\% près en incertitude relative.

L'analyse des trois premières années de données de SNLS (250 SN Ia), en cours d'achèvement, conclut dans le même sens, mais avec une précision accrue. La densité de matière est maintenant contrainte avec une incertitude relative de $10 \%$, et la densité d'énergie noire est constante dans le temps, à $6 \%$ près en incertitude relative. Affiner la précision de ce dernier test est l'un des objectifs majeurs de la cosmologie observationnelle actuelle, car une densité d'énergie noire constante dans le temps favoriserait une interprétation en termes de constante cosmologique.

\section{Mieux connaître les supernovæ de type Ia}

Même si le mécanisme supposé à l'origine des SN Ia conduit à une homogénéité de leurs propriétés spectrales et lumineuses, les courbes de lumière mesurées et corrigées du décalage spectral montrent une dispersion d'environ 30\%. D'une supernova à l'autre, ces courbes sont donc proches, mais ne deviennent superposables qu'après une correction empirique, fonction de deux caractéristiques intrinsèques des SN Ia. Ces caractéristiques sont liées à la durée de l'émission de lumière et au rapport des flux émis dans deux bandes de fréquence de référence. Ce sont les courbes de lumière corrigées de ces effets de dispersion intrinsèque qui sont utilisées pour les mesures cosmologiques. À l'heure actuelle, la même correction est utilisée quel que soit le décalage spectral, car on suppose que les SN Ia sont les mêmes, qu'elles soient proches (explosions récentes) ou lointaines (explosions anciennes).

Grâce à la statistique élevée et à la qualité des données de SNLS, on peut tester la validité de cette hypothèse en vérifiant si les caractéristiques changent entre SN Ia lointaines et proches, ou s'il existe des sous-lots de SN Ia aux caractéristiques différentes. De nombreuses études ont été réalisées en ce sens sur la base des 250 SN Ia collectées sur trois ans ([2] à [7]). Aucun effet significatif n'a été relevé, à l'exception d'une faible différence entre les SN Ia explosant dans les galaxies âgées, qui ne forment plus d'étoiles, et celles explosant dans les galaxies jeunes, actives sur le plan de la formation stellaire [4]. Les SN Ia des galaxies âgées ont en effet des courbes de lumière intrinsèques plus rapides, moins lumineuses et plus rouges que celles qui explosent dans un environnement jeune. Cet effet induit une faible variation des caractéristiques moyennes des SN Ia en fonction du décalage spectral [5], car les proportions de SN Ia dues aux galaxies jeunes et âgées varient avec ce dernier [4]. Cependant, au niveau de précision actuel, cette variation n'a pas d'impact sur les résultats cosmologiques : la modélisation actuelle des effets de dispersion intrinsèque reste donc valide. Les deux dernières années de données de SNLS devraient nous en apprendre un peu plus.

Mais pour aller au-delà, il faudra attendre le démarrage vers 2015 des projets actuellement en cours de construction, dont l'objectif est de détecter des centaines de milliers de supernovæ Ia : il sera alors possible de mieux comprendre les différences entre SN Ia et de contrôler leur impact éventuel sur les résultats cosmologiques [8]. Nul doute que ces projets, combinés aux autres méthodes cosmologiques, comme la mesure des oscillations acoustiques baryoniques, des effets de lentilles gravitationnelles faibles ou le comptage des amas de galaxies, nous feront faire un pas de géant dans la compréhension de ce qui se cache derrière l'énergie noire [9]. 